\title{
Filigrane
}

Écoutes psychothérapiques

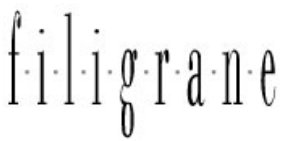

\section{Quelle langue pour la Shoah ?}

\section{David Benhaïm}

Volume 21, numéro 1, printemps 2012

URI : https://id.erudit.org/iderudit/1012882ar

DOI : https://doi.org/10.7202/1012882ar

Aller au sommaire du numéro

\section{Éditeur(s)}

Revue Santé mentale au Québec

\section{ISSN}

1192-1412 (imprimé)

1911-4656 (numérique)

Découvrir la revue

\section{Citer cet article}

Benhaïm, D. (2012). Quelle langue pour la Shoah ? Filigrane, 21(1), 65-88. https://doi.org/10.7202/1012882ar

\section{Résumé de l'article}

L'auteur montre que la langue est un élément de la culture auquel les totalitarismes du xx${ }^{\mathrm{e}}$ siècle se sont attaqués de façon délibérée. Ils ont essayé d'intoxiquer la langue ou d'en forger une nouvelle, le novlangue, pour manipuler et influencer les masses dans le sens de leurs intérêts, pour empêcher toute expression d'une pensée critique. La propagande et l'idéologie ont été les moyens qu'ils ont utilisés pour le faire. Le pouvoir absolu s'est chargé d'imposer des mots, des tournures, des expressions, des énoncés qu'il n’a cessé de marteler tout en éliminant d'autres. Cet excès de violence était destiné à empêcher tout travail de subjectivation en rendant impossible la constitution d'un espace psychique propre, condition essentielle de la subjectivation. Dans cette perspective, l'auteur se pose la question du rapport entre cette perversion de la langue et l'apparition des Lagers et des Goulags. La plupart des écrivains qui ont survécu à l'expérience concentrationnaire ont abordé la question de la langue comme s'il s'agissait d'une question incontournable. À travers l'analyse et le commentaire de deux auteurs, Primo Levi et Imre Kertèsz, et de leur réflexion sur la langue des camps et du système totalitaire, l'auteur tente d'ouvrir des pistes de réflexion sur ce qui, dans la question, demeure obscur et énigmatique.
Ce document est protégé par la loi sur le droit d'auteur. L'utilisation des services d’Érudit (y compris la reproduction) est assujettie à sa politique d'utilisation que vous pouvez consulter en ligne.

https://apropos.erudit.org/fr/usagers/politique-dutilisation/ 


\title{
Quelle langue pour la Shoah?
}

\author{
David Benhaïm
}

L'auteur montre que la langue est un élément de la culture auquel les totalitarismes du $x x^{\mathrm{e}}$ siècle se sont attaqués de façon délibérée. Ils ont essayé d'intoxiquer la langue ou d'en forger une nouvelle, le novlangue, pour manipuler et influencer les masses dans le sens de leurs intérêts, pour empêcher toute expression d'une pensée critique. La propagande et l'idéologie ont été les moyens qu'ils ont utilisés pour le faire. Le pouvoir absolu s'est chargé d'imposer des mots, des tournures, des expressions, des énoncés qu'il n'a cessé de marteler tout en éliminant d'autres. Cet excès de violence était destiné à empêcher tout travail de subjectivation en rendant impossible la constitution d'un espace psychique propre, condition essentielle de la subjectivation. Dans cette perspective, l'auteur se pose la question du rapport entre cette perversion de la langue et l'apparition des Lagers et des Goulags. La plupart des écrivains qui ont survécu à l'expérience concentrationnaire ont abordé la question de la langue comme s'il s'agissait d'une question incontournable. À travers I'analyse et le commentaire de deux auteurs, Primo Levi et Imre Kertèsz, et de leur réflexion sur la langue des camps et du système totalitaire, l'auteur tente d'ouvrir des pistes de réflexion sur ce qui, dans la question, demeure obscur et énigmatique.

\section{Introduction}

es totalitarismes du Xx $\mathrm{X}^{\mathrm{e}}$ siècle pourraient être étudiés comme des phéno_ mènes de nature linguistique. La langue est un élément de la culture auquel ils se sont attaqués de façon délibérée. Certains ont essayé de forger une nouvelle langue, le newspeak ou novlangue tel que la désigne Orwell. D'autres ont essayé, comme le pense Victor Klemperer, d'infecter la langue. Ainsi les nazis ont inoculé un poison à la langue allemande. Dans son livre LTI, la langue du troisième Reich, il écrit:

Le nazisme s'insinua dans la chair et dans le sang du grand nombre, à travers des expressions isolées, des tournures, des formes syntaxiques qui s'imposaient à des millions d'exemplaires et qui furent adoptées de façon mécanique et inconsciente. (Klemperer, 1996, 40) 
Mais d'abord qu'est-ce qu'on entend par langage totalitaire?

Le langage totalitaire est l'usage qu'un pouvoir absolu fait du langage à des fins politiques: propagande, idéologie. (Dewitte, 2007, 11) Si nous suivons Saussure dans sa distinction entre langue et parole, nous dirons que la langue est un système de signes permettant aux individus de communiquer entre eux, alors que la parole est le libre usage que chacun d'entre nous fait de sa propre langue. Si nous restons dans ce cadre saussurien, nous pouvons affirmer que la propagande et l'idéologie, moyens qu'utilise le pouvoir absolu pour manipuler et influencer les masses dans le sens de ses intérêts, pour modeler l'opinion, sont un fait de parole. En ce sens nous pouvons qualifier cette parole d'idéologique et souligner que le pouvoir va se charger d'imposer des mots, des tournures, des expressions, des énoncés qu'il va marteler tout en éliminant d'autres. La langue va se modifier en conséquence. C'est ainsi qu'un régime politique va exercer une «emprise [...] sur l'esprit, sur la sensibilité, voire sur la réalité» (ibid., 11). Certes la langue est toujours contraignante et apprendre à la parler constitue ce que Castoriadis-Aulagnier appelle la violence primaire, violence radicale et nécessaire (CastoriadisAulagnier, 1975, 38). Cependant les changements qu'un régime politique introduit dans les termes et les expressions, les modifications qu'il introduit dans la langue, constituent une violence secondaire, un excès de violence «le plus souvent nuisible et jamais nécessaire au fonctionnement du Je, malgré la prolifération et la diffusion dont il fait preuve» (ibid., 38).

Il institue un en plus de contrainte pour l'usager de la langue dont la parole ne sera plus tout à fait «libre usage»; il va ainsi transformer insensiblement sa représentation de lui-même et de la réalité. La parole est alors aliénée dans la mesure où le sujet parlant est assujetti à un code qui l'enferme dans certaines limites le contraignant à penser et à interpréter les événements, la réalité dans une certaine perspective. C'est ce que Klemperer et Orwell montreront à profusion.

Si la fiction permet de penser la réalité et de dégager des dimensions qu'autrement nous aurions de la peine à concevoir, nous pouvons dire que le roman fiction 1984 permet de comprendre les transformations que les totalitarismes du $\mathrm{xx}^{\mathrm{E}}$ siècle ont fait subir à la langue. Le projet du novlangue qu'Orwell présente nous aide à saisir les intentions qui président à ces transformations. Il s'agit d'abord et avant tout de créer un langage qui empêche de concevoir et de dire toute pensée que le Pouvoir considère comme non orthodoxe, de nommer toute réalité interdite. Le sujet parlant est alors réduit à ne pouvoir penser ni dire que ce qui est. 
Le distique de Schiller, «la langue cultivée qui poétise et pense à ta place » (Klemperer, 1996,40), constitue un des axes qui oriente la réflexion de Klemperer. On a coutume de le citer d'un point de vue purement esthétique comme quelque chose d'anodin, cependant, nous fait remarquer Klemperer, la langue ne fait pas que poétiser et penser à ma place,

Elle dirige aussi mes sentiments, elle régit tout mon être moral d'autant plus naturellement que je m'en remets inconsciemment à elle. (Klemperer, 1996, 40)

Et Klemperer de poser la question qui est au cœur de la langue totalitaire:

Et qu'arrive-t-il si cette langue cultivée est constituée d'éléments toxiques? Les mots peuvent être comme de minuscules doses d'arsenic: on les avale sans y prendre garde, ils semblent ne faire aucun effet, et voilà qu'après quelque temps l'effet toxique se fait sentir. (Klemperer, 1996, 40)

Cette question est d'autant plus pertinente que la langue est une institution qui nous précède: à travers l'acquisition de la langue maternelle s'opère une transmission de manières de percevoir la réalité, de la sentir, de manières de penser et une transmission de valeurs.

L'acquisition du langage, fait remarquer le linguiste É. Benveniste, est une expérience qui va de pair chez l'enfant avec la formation du symbole et la construction de l'objet. Il apprend les choses par leur nom; il découvre que tout a un nom et que d'apprendre les noms lui donne la disposition des choses. Mais il découvre qu'il a lui-même un nom et que par là il communique avec son entourage. Ainsi s'éveille en lui la conscience du milieu social où il baigne et qui façonnera peu à peu son esprit par l'intermédiaire du langage. À mesure qu'il devient capable d'opérations intellectuelles plus complexes, il est intégré à la culture qui l'environne. (Benveniste, 1966, 2930)

Dans cette perspective, le distique de Schiller acquiert un sens radical, comme le souligne Dewitte (Dewitte, 2007,166) lorsqu'on l'insère dans le contexte de la langue du Troisième Reich.

Cette dernière est parvenue à son degré de toxicité le plus haut en défigurant certains mots, en modifiant leur valeur - transformation du 
péjoratif en laudatif -, en créant des euphémismes, des néologismes, des mots réducteurs, etc., et, finalement, en favorisant leur emploi plus fréquent. Ainsi

elle assujettit la langue à son terrible système, elle gagne avec la langue son moyen de propagande le plus puissant, le plus public et le plus secret. (Klemperer, 1996, 41).

Le projet qui se cache derrière cette transformation de la langue est de renforcer l'idéologie en empêchant la possibilité de toute pensée critique : il s'agit d'idéologiser la pensée de façon à l'empêcher de concevoir ce que le Pouvoir ne veut pas qu'elle conçoive. Ce projet repose sur le postulat selon lequel la pensée naît avec le langage, qu'elle lui est complètement subordonnée. Si on modifie la langue de quelque façon que ce soit, il s'ensuivra une modification de la pensée. La pensée ne peut penser que ce que la langue lui permet de penser. C'est à partir de là que commence l'idéologisation. En effet, la modification se fera en fonction des pensées qu'on veut supprimer. Empêcher de concevoir certaines idées ce serait faire disparaître certaines réalités. En poussant les choses à l'extrême, nous dirons que si le sens des mots est fixé une fois pour toutes, la dimension métaphorique de la langue est ainsi éliminée. Autrement dit, les mots auront, par exemple, un sens propre mais pas de sens figuré, ce qui enferme l'esprit dans une réalité unidimensionnelle. Les choses se définiront par leur fonction ; la pensée deviendra alors opératoire. On s'attaque ainsi au pouvoir fondateur de la langue. Nous pouvons à nouveau convoquer Benveniste qui écrit:

L'homme a toujours senti - et les poètes ont souvent chanté - le pouvoir fondateur du langage, qui instaure une réalité imaginaire, anime les choses inertes, fait voir ce qui n'est pas encore, ramène ici ce qui a disparu. C'est pourquoi tant de mythologies, ayant à expliquer qu'à l'aube des temps quelque chose ait pu naître de rien, ont posé comme principe créateur du monde, cette essence immatérielle et souveraine, la Parole. (Benveniste, 1966, 25)

On pourrait légitimement se demander quel intérêt, sinon historique, peut avoir une telle interrogation sur la langue maintenant que les totalitarismes semblent, apparemment, avoir disparu. La réponse serait que la langue ne saurait retrouver son état initial. Elle restera marquée par l'expérience totalitaire. Dans un ouvrage aujourd'hui oublié mais qui a fait rage en Mai 68, L'homme unidimensionnel (1968), Herbert Marcuse s'est livré à une analyse du nov- 
langue de nos sociétés industrielles et post-industrielles. Il souligne que l'univers de la communication dans lequel s'exprime le comportement unidimensionnel est façonné par des agents de publicité. Son langage témoigne de l'identification et de l'unification, de la promotion systématique de la pensée positive et de l'action positive, de l'attaque concertée contre les notions critiques et transcendantes. Dans les modes de langage prévalents, existe

un contraste entre les formes de pensée dialectiques, bi-dimensionnelles, et le comportement technologique ou les «habitudes de pensée» sociales. [...] La parole et le langage s'imprègnent d'éléments magiques, autoritaires, rituels. (Marcuse, 1968, 110)

L'écart et la tension qui existent entre apparence et réalité, entre cause et effet, entre substance et attribut, tendent à disparaître complètement. La désignation, l'assertion et l'imitation prennent le pas sur les éléments d'autonomie, de découverte, de démonstration et de critique qui finissent par s'estomper. Le discours est privé des médiations qui constituent les étapes du processus de connaissance et d'évaluation cognitive. Les concepts qui permettent de saisir les faits et par là même les transcender, «perdent leur représentation linguistique authentique. » Sans ces médiations, le langage tend à exprimer et à promouvoir

l'identification immédiate entre le facteur et le fait, entre la vérité et la vérité établie, entre l'essence et l'existence, entre la chose et sa fonction. (Marcuse, 1968, 110. Ce sont mes italiques)

Je traduirais cette analyse marcusienne en langage psychanalytique contemporain en affirmant que ces sociétés totalitaires et leurs novlangues s'attaquent aux processus de subjectivation. Cette notion, introduite par Raymond Cahn (1991) qui a largement contribué à sa diffusion, est intimement liée à celle de langage et de parole. Deux idées clés sont à retenir lorsque nous parlons de subjectivation: d'abord celle d'appropriation subjective et de transformation où non seulement ce qui était refoulé mais aussi ce qui était clivé est réapproprié et transformé, ensuite l'idée que la subjectivation est constituée par un ensemble de processus à l'œuvre tout au long de l'existence, mais qui demeurent inachevés. La subjectivation est mise à mal par les langages totalitaires parce que leur fonction politique et idéologique est, comme le montre Orwell, «non [d']étendre mais [de] diminuer le domaine de la 
pensée » en réduisant au minimum le choix des mots. En ce sens, le novlangue semble destiné à empêcher tout travail de subjectivation et, à la limite, à provoquer, à la longue, la mort du sujet. Il rend impossible la constitution d'un espace psychique propre, condition essentielle de la subjectivation. Instrument privilégié de la propagande politique, son intrusion continuelle finit par envahir et sidérer le psychisme détruisant ainsi toutes les conditions d'une parole propre. Langage d'action et non de représentation comme le montre Marcuse, le dire reste assujetti à l'agir et à l'efficacité immédiate. Comme j'aurai l'occasion de le développer plus longuement dans ce texte, non seulement ces langages totalitaires empêchent la subjectivation mais ils sont à la source de la désubjectivation du sujet.

Ce sombre tableau, quelles que soient les réserves que l'on puisse avoir face à l'analyse marcusienne, fait de nous les héritiers linguistiques des sociétés totalitaires. En tant que psychanalystes, nous ne pouvons pas rester indifférents face à cette dégradation du langage dans la mesure où elle s'accompagne d'une détérioration des capacités d'élaboration du sujet. Le langage est l'âme même de notre discipline et tout ce qui s'attaque à lui compromet l'exercice même de la psychanalyse. Il est essentiel de rappeler que les conditions politiques de l'exercice et du développement de la psychanalyse sont liées à la démocratie et toute société de type totalitaire met en danger son existence dans la mesure où la parole s'aliène dans des crédos et des interdictions qui n'admettent pas d'être interrogés. La terreur qui s'installe transforme les analystes en ennemis intérieurs qu'on persécute comme ce fut le cas dans l'Allemagne nazie ou plus près de nous, en Argentine, au cours de la dernière dictature.

Cela me conduit à poser une question qui pourrait sembler incongrue au premier abord: quels liens peuvent bien exister entre cette perversion de la langue et l'apparition des Lagers et des Goulags? Il est frappant de constater que la plupart des écrivains qui ont survécu à l'expérience concentrationnaire - Jean Améry, Primo Levi, Aharon Appelfeld, Paul Celan, pour ne citer que ceux-là - ont à un moment ou à un autre de leur réflexion abordé le problème de la langue comme si la question était incontournable. Je convoquerai deux auteurs qui nous ont laissé un témoignage de leur passage par les camps de concentration pour lesquels la question de la langue est au centre de l'expérience concentrationnaire: Primo Levi et Imre Kertèsz. Leur réflexion sur le langage nous permettra non de répondre à la question posée mais d'ouvrir des pistes de réflexion sur ce qui, dans la question, demeure obscur et même énigmatique. 


\section{Primo Levi}

Dans Conversations avec Primo Levi, Ferdinando Camon lui exprime l'impression ressentie à la lecture de ses livres: «On sent un écrivain doué de fantaisie, à la langue pleine de vie, débordante de métaphores. " (Camon, 1991, 70) Primo Levi lui répond: «La question de la langue a commencé à m'intéresser de plus en plus à mesure que j'avançais dans mon œuvre d'écrivain.» (Camon, 1991, 70)

Dans Retour à Auschwitz, transcription d'un entretien télévisé réalisé en 1982, il déclare: «À mon avis, parmi les causes premières de tant de naufrage dans le Camp, la langue, le langage figurait en tête.» (Levi, 2005, 106)

\section{Langue et témoignage}

Dans son livre Les naufragés et les rescapés (Levi, 1989, 87-103), Levi aborde le problème de la langue par le biais de la communication. Le besoin de communiquer est vital chez lui : «Je suis un parleur, dit-il à Ferdinando Camon. Si l'on me ferme la bouche, je meurs. Et là, on me fermait la bouche.» (Camon, 1991,54)

Il commence par s'insurger contre le terme d'« incommunicabilité» à la mode dans les années soixante-dix, le qualifiant de «monstre linguistique». Incommunicabilité signifie, pour lui, que le langage courant ne saurait traduire nos pensées les plus profondes et qu'en conséquence, il ne ferait que les trahir. Il souligne cependant l'insuffisance du langage qui a été créé pour des êtres qui vivent dans un monde «normal», libre, à l'abri des expérienceslimites jamais vues ni avant ni après ni ailleurs. Ainsi dans le chapitre 13 de son livre Si c'est un homme, il soulève un des problèmes essentiels qui se posent au témoin en rapport avec la langue: comment faire part au lecteur de son témoignage lorsque les mots n'ont pas le même sens pour les deux? Les mots «faim», «froid» disent autre chose,

des choses que ne peuvent exprimer les mots libres, créés par et pour des hommes libres qui vivent dans leur maison et connaissent la joie et la peine.» (Levi, 1987, 132)

\section{Comment alors transmettre?}

Si les Lager avaient duré plus longtemps, ils auraient donné le jour à un langage d'un âpreté nouvelle, celui qui nous manque pour expliquer ce que c'est que peiner tout le jour dans le vent, à une température au-dessous de zéro, 
avec, pour tous vêtements, une chemise, des caleçons, une veste et un pantalon de toile, et dans le corps la faiblesse et la faim, et la conscience que la fin est proche. (Levi, 1987, 132.Ce sont mes italiques)

Cette langue dont parle Lévi n'existe pas. C'est pourquoi comme écrivain il choisira le parti pris de la clarté pour reprendre le titre du livre que Françoise Carasso lui consacre. Précision, méthode, clarté et sobriété sont des caractéristiques de son style, expression peut-être de sa formation scientifique, mais choix éthique et politique face au mensonge et à la destruction que les nazis ont exercé sur le langage. Il essaiera ainsi par son écriture de traduire ce que seule cette nouvelle langue aurait pu exprimer.

\section{Langue et Lager}

S'il est vrai, comme l'affirme Benveniste, que «seule la langue permet la société, qu'elle constitue ce qui tient ensemble les hommes, le fondement de tous les rapports qui à leur tour fondent la société» (Benveniste, 1974, 54), nous comprenons pourquoi Primo Levi a eu recours à l'histoire biblique de la tour de Babel pour décrire le chaos linguistique du Lager. Le narrateur biblique donne la parole à Dieu qui s'exclame:

«Allons! Descendons et ici même, confondons leur langage, en sorte qu'ils ne comprennent plus le langage les uns des autres.» (La Bible, Ancien Testament I, 1956, 35)

Le Lager, c'est la destruction du lien social, c'est la dispersion des hommes comme dans l'épisode biblique où Dieu les punit en rendant impossible toute communication entre eux. C'est la discorde, le tohu-bohu, l'incompréhension. De plus, dans ce contexte, la langue devient quelque chose d'hostile et d'étranger. Levi raconte comment les détenus se réveillent au milieu de la nuit, « sous le coup d'un ordre, crié par une voix haineuse, et dans une langue que nous ne comprenons pas» (Levi, 1987, 66-67).

Bien des années après sa sortie du camp, il continuera d'entendre, en polonais ou en allemand, ce mot: debout! Jean Améry - anagramme de Hans Mayer -, de son côté, dans Par-delà le crime et le châtiment, montre comment le travail de la pensée, la vie de l'esprit, disparaissent lorsque l'appartenance sociale est ainsi détruite et qu'on ne dispose plus d'une langue commune. Dans La survivance (Altounian, 2000,123), Janine Altounian analyse très finement le texte d'Améry et va jusqu'à parler de meurtre de la langue. 
Primo Levi parle du «heurt contre la barrière linguistique» (Lévi, 1989, 89), du fait que «savoir l'allemand ou pas était une ligne de partage» (Levi, 1989, 90). Il décrit la férocité des SS dans la profération d'un ordre dans un crescendo qui monte et éclate finalement en une pluie de coups: il était d'abord prononcé d'une «voix paisible», puis répété d'une «voix plus haute et furieuse ", "hurlé à plein gosier » comme lorsqu'on s'adresse à un animal domestique, "plus sensible au ton qu'au contenu du message» (Levi, 1989, 90). Ceux qui hésitaient parce qu'ils ne comprenaient pas étaient roués de coups. Levi souligne que les coups étaient « une variante du même langage: l'usage de la parole pour communiquer la pensée, ce mécanisme nécessaire et suffisant pour que l'homme soit homme, était tombé en désuétude.» (Levi, $1989,90)$

Quand l'usage de la parole connaît une éclipse, nous sommes déjà dans le monde animal, sur le chemin de la déshumanisation. Le hurlement ou le coup de poing sont des signes comparables aux piqûres des éperons, au claquement du fouet ou aux tapes sur l'échine dont on se sert pour dresser un cheval. Citant le livre de Marsalek, Mauthausen, Levi rapporte que dans ce camp plus multilingue qu'Auschwitz, la matraque en caoutchouc "était appelée der Dolmetscher: «l'interprète », celui qui se faisait comprendre par tous. » (Levi, 1989, 90)

Marie Moscovici évoque les insultes échangées entre les guerriers, dans L'Iliade, « en guise de blessures non infligées par les armes» (Moscovici, 2002, 29) pour les comparer aux considérations de Primo Levi sur les coups qui, dans les camps, tenaient lieu de langage: «Tous les kapos nous frappaient: [...] c'était leur langage.» (Levi, 1989, 72)

Ce que Levi commente ainsi :

En effet, l'homme inculte (et les Allemands de Hitler, et les SS en particulier, étaient terriblement incultes : ils n'avaient pas été "cultivés», ou avaient été mal cultivés) est incapable de distinguer entre celui qui ne comprend pas sa langue et celui qui ne comprend pas tout court. (Levi, 1989, 91)

Les nazis étaient convaincus - c'était une conviction essentielle de leur idéologie - qu'il n'existait qu'une seule civilisation, la leur. Toute autre n'avait de valeur que dans la mesure où elle pouvait contenir en elle quelque élément de germanité, autrement elle était renvoyée à la barbarie. Toute langue autre que l'allemand, fait remarquer Levi, était considérée comme une non-langue, et celui qui la parlait, « il fallait le faire taire avec des coups et le remettre à sa 
place, à tirer, porter et pousser, puisqu'il n'était pas un Mensch, un être humain.» (Levi, 1989, 91. Ce sont mes italiques.)

Il s'ensuit que, dans l'impossibilité d'adresser la parole à ceux qui vous hurlent ou vous frappent, "votre langue se dessèche [...] et, avec la langue, la pensée.» (Levi, 1989, 92)

À travers un cours particulier et accéléré d'allemand, payable en pain, qu'un détenu Alsacien acceptera de lui donner entre le moment du couvrefeu et le sommeil, Primo Levi fera la découverte que

l'allemand du Lager, squelettique, hurlé, constellé d'obscénités et d'imprécations, n'avait qu'une vague parenté avec la langue précise et austère de mes livres de chimie et avec l'allemand mélodieux et raffiné des poésies de Heine que me récitait Clara, une camarade d'études (Levi, 1989, 96).

Le langage du Lager est un «Lagerjargon », langage sectoriel, «étroitement apparenté avec le vieil allemand des casernes et avec le nouvel allemand des SS » (Levi, 1989,97), mais également influencé par les diverses langues parlées dans le Lager.

Il fera plus tard le lien avec l'ouvrage de Klemperer, LTI, La langue du Troisième Reich, et verra dans cet allemand du Lager, « une langue spéciale [...] une variante barbare» (Levi, 1989, 96) de la LTI. Il en conclura que « [1]à où l'on fait violence à l'homme, c'est une observation évidente, on le fait aussi à la langue.» (Levi, 1989, 96)

Je voudrais en terminant cette analyse rapporter un événement qui pourrait paraître anecdotique, mais qui traduit la volonté de Primo Levi de faire connaître la langue d'Auschwitz: l'attention qu'il a apportée à la traduction en allemand de son livre, Si c'est un homme. Dans son livre Primo Levi ou la tragédie d'un optimiste, Myriam Anissimov nous parle de la relation que Levi entretenait avec son traducteur autour de ce travail:

Bien que Riedt [le traducteur] fût un fin lettré, il ne connaissait pas - et pour cause - le jargon immonde des camps d'extermination. Son allemand était beaucoup plus distingué que celui que Levi avait appris à Auschwitz. Aussi chaque mot fut-il longuement pesé et discuté, après que Levi eut communiqué les phrases dont il avait conservé le souvenir. La plupart du temps Heinz Riedt lui faisait remarquer que ce n'était pas là du bon allemand, et Levi lui répondait que c'était pourtant ainsi qu'on parlait à Auschwitz. Il voulait que rien ne fût perdu de cette langue allemande qui avait subi une mutation dans les camps de la mort. (Anissimov, 1996, 537) 


\section{Imre Kertèsz ou la langue exilée}

Il est important de situer Kertèsz en rappelant brièvement qu'il a connu les deux totalitarismes du $\mathrm{Xx}^{\mathrm{e}}$ siècle et en a été profondément marqué. Déporté en 1944, à l'âge de quinze ans, il a été à Auschwitz, puis Buchenwald et Zeitz. De retour en Hongrie, il a vécu quarante ans sous le régime communiste.

Sa réflexion sur la langue a comme toile de fond un bref récit du poète Paul Celan, Entretien dans la montagne, dont il commente librement certaines parties et sur lequel il revient, à plusieurs reprises, au cours de sa réflexion. Paul Celan, de son vrai nom Paul Pessakh Antschel, est lui-même un survivant de la Shoah. En juillet 1942, Il sera interné dans un camp de travail à Tabaresti, en Moldavie, après avoir perdu ses parents. Son œuvre poétique ne saurait être dissociée de la Shoah; elle est fondée sur Auschwitz comme l'ont souligné la plupart de ses commentateurs. En juillet 1959, Celan devait rencontrer le philosophe Theodor W. Adorno à Sils Maria. La rencontre n'a pas eu lieu. En août 1959, comme il l'évoque dans Le Méridien, il composa l'Entretien dans la montagne:

Et voici un an, remémoration d'une rencontre manquée dans l'Engadine, j'ai porté au net un bref récit où, à l'instar de Lenz, j'ai ouvert à quelqu'un chemin en montagne. Je m'étais, l'une et l'autre fois, à partir d'un « 20 janvier », le mien, sur tel pas transcrit. Une rencontre m’a mis en présence de... moi-même. (Celan, 2008, 40)

Dans son remarquable commentaire, Stéphane Mosès présente ainsi l'Entretien dans la montagne:

Chemin de moi vers moi, l'Entretien dans la montagne accomplit, sur l'horizon d'une absence, d'un manque, d'une rencontre qui n'a pas eu lieu, un trajet à travers la forêt des mots, trajet au cours duquel un langage anonyme se transforme peu à peu en parole de sujet, un Il en Je et Tu, un récit en discours. Le thème de ce texte c'est sa forme même: la conversion de l'impersonnel en personnel, l'émergence du sujet parlant hors de ses divers masques. (Celan, 2001, 26)

Autrement dit, le texte est le récit de la subjectivation, de la naissance de la subjectivité du poète. Le premier passage que cite Kertèsz pose une question : que possède le Juif qui lui appartienne en vérité? 
Un soir que le soleil, pas lui seulement, avait sombré, [...] le Juif s'en fut sous les nuages, s'en fut dans l'ombre, la sienne et l'étrangère - car le Juif, tu le sais bien, que possède-t-il, qui lui appartienne en vérité, qui ne soit pas emprunté... (Entretien dans la montagne, trad. John E. Jackson et André du Bouchet, éd. Fata Morgana, in Kertèsz, 2009, 213)

Kertèsz répond: seul Auschwitz lui appartient, mais «par Auschwitz, par cette terrible propriété, il a perdu sa langue.» (Kertèsz, 2009, 214) Affirmation qui prendra tout son sens dans la suite de sa réflexion. Qu'est-ce que la perte de la langue? Kertèsz souligne la particularité d'un phénomène propre aux dictatures du XX $\mathrm{X}^{\mathrm{e}}$ siècle: le novlangue, phénomène sans précédent dans l'histoire.

Pour lui, cette langue exclut l'individu de sa propre vie intérieure et par là même de sa propre langue dans la mesure où sont éliminés les mots et les expressions qui permettraient d'identifier et de dire ce qui naît et se développe en nous. Le processus implique « une dynamique bien dosée de violence et de terreur» (ibid., 215) qui fait qu'elle "pénètre irrésistiblement la conscience de l'individu» (Kertèsz, 2009, 215). Il en résulte une identification de l'individu au rôle qu'on lui impose, qu'il corresponde ou non à sa personnalité. Il ne saurait survivre qu'en acceptant totalement ce rôle. Mais cette survie est en même temps l'anéantissement de sa personnalité. Il devient la proie du langage anonyme, impersonnel, désubjectivé, dont parle Celan. Cette rapide description nous place au cœur de la désubjectivation si par désubjectivation on entend la perte de la capacité à se vivre comme sujet dans toute situation qui vient désorganiser la vie psychique du sujet. En effet, les traumas, les catastrophes psychiques, résultat de bouleversements sociaux, de guerres ou de génocides, constituent des menaces imparables de désubjectivation, en détruisant les conditions de l'intersubjectivité et de l'espace où le Je peut advenir, "espace parlant», pour reprendre l'expression de Piera Aulagnier. Il est essentiel, dans notre contexte, de préciser que le Je est une instance constituée par le discours. S’il est vrai, comme le souligne encore Aulagnier, qu'il existe des "conditions nécessaires pour que cet espace offre au Je un habitat conforme à ses exigences » (Castoriadis-Aulagnier, 1975, 129), le novlangue crée, au contraire, les conditions de son aliénation en excluant du langage « tout mot, toute signification, tout concept nécessaires à une mise en parole » (Aulagnier, 1984, 239). Si l'on peut considérer la subjectivation comme un processus psychique toujours à l'œuvre, la désubjectivation peut à son tour être considérée comme ce qui menace continuellement le sujet. Les totalitarismes du Xx ${ }^{\mathrm{e}}$ et du XXI ${ }^{\mathrm{e}}$ siècles ont détruit ce que Hannah 
Arendt appelle le vivre ensemble lui substituant les mouvements de masse où, comme Freud nous l'a montré, le sujet se dissout. Au chapitre IX de Psychologie des masses et analyse du moi, il souligne un des caractères les plus frappants de la masse: «le manque d'autonomie et d'initiative chez l'individu, la similarité de sa réaction avec celle de tous les autres, pour ainsi dire sa réduction au rang d'individu de la masse» (Freud, 1921, 55).

Freud développe, sans la nommer, cette notion de désubjectivation et les conditions qui l'instaurent, lorsqu'au chapitre VIII, il compare la relation hypnotiseur/hypnotisé à l'état amoureux mais aussi à la relation du meneur à la masse. «La relation hypnotique est, écrit-il - si cette expression est permise - une formation en masse à deux. » (Freud, 1921, 53) Dans tous ces cas le sujet perd sa qualité de sujet pour se fondre dans une masse homogène qui se caractérise par une indifférenciation des sujets qui la constituent et par la perte de la limite psychique individuelle. L'homogénéité est mentale et affective. Le meneur est à la masse, ce que l'hypnotiseur est à l'hypnotisé, l'analogie illustre le rôle capital du meneur. Il hypnotise la masse. La structure psychologique de la masse se constitue lorsqu' «un certain nombre d'individus [...] ont mis un seul et même objet à la place de leur idéal du moi et se sont, en conséquence, identifiés les uns aux autres dans leur moi» (54). Cette somme d'individus identifiés les uns aux autres est une somme d'individus désubjectivés dont les traits principaux sont «l'affaiblissement du rendement intellectuel, et la non-inhibition de l'affectivité, l'inaptitude à la modération et à l'ajournement, le penchant à l'outrepassement de toutes les barrières dans la manifestation de sentiment et à la pleine éconduction de celle-ci dans l'action» (55).

Dans les premières pages de Journal de galère, écrit entre 1961 et 1991, Kertèsz s'interroge sur les possibilités de l'art à une époque où le type tragique, qui a toujours été sa source d'inspiration, n'existe plus.

Le héros tragique, écrit-il, est un homme qui se crée lui-même et qui échoue. Or, de nos jours, il ne fait plus que s’adapter. (Kertész, 1992, 2010, 11.)

Le héros tragique est un sujet qui se construit, qui se crée lui-même. Même si son destin est d'échouer, il est porteur de cette capacité à subjectiver que l'on reconnaît comme l'instrument et le résultat du travail analytique. Ce destin - que l'on peut comprendre aujourd'hui comme constitué par les conflits inconscients et les forces pulsionnelles qui l'habitent - était conçu 
autrefois comme une puissance extérieure qui régissait l'univers et le cours des événements. En revanche, l'homme moderne est l'homme fonctionnel, autrement dit désubjectivé. Nous pourrions le rapprocher du sujet en état opératoire, tel que les psychosomaticiens de l'École de Paris l'ont décrit. Ce qui le caractérise au plus haut point, c'est « une non-pensée», selon l'expression de Claude Smadja. Ce dernier, dans son livre La vie opératoire, esquisse un rapprochement pertinent entre l'activité psychique d'un individu de masse tel que Freud le décrit et un sujet en état opératoire:

Chez l'un comme chez l'autre, la pensée a perdu ses qualités individuelles, ce qui en faisait sa singularité, son unicité. Elle est devenue une pensée aux ordres du collectif. Elle est devenue une pensée collective conforme à un même et unique modèle. Une non-pensée. (Claude Smadja, 2001 188-189)]

Il est aliéné, selon Kertèsz. Il a renoncé à la réalité, à l'existence. Il vit dans une pseudo réalité, une vie qui se substitue à la vie comme la fonction s'est substituée à lui. Sa vie est une erreur tragique mais sans les conséquences ni les antécédents tragiques nécessaires, car les conséquences, dans la tragédie classique, étaient le résultat de la loi propre aux caractères et aux actes. À l'époque moderne, elles sont imposées par la nécessité de l'équilibre du système social - toujours absurde du point de vue de l'individu, selon Kertèsz. La vie individuelle est déterminée d'avance et ne doit occuper que l'emplacement qui lui a été assigné. Dans un entretien réalisé par Carola Hähnel et Philippe Mesnard et publié par la revue Mouvements, Kertèsz précisera que ce type d'homme qui vit sous le totalitarisme, qu'il a souvent " décrit et tenté de saisir philosophiquement [...] c'est un personnage non-psychologique qu'on ne peut pas du tout comprendre en termes psychologiques» (117), il s'agit plutôt d'un homme plongé dans une expérience de masse dans laquelle l'individu se dissout. Pour reprendre le titre de son premier roman, nous dirons que l'individualité est supprimée et que nous avons affaire à un «être sans destin ». Selon Kertèsz, cela tient à la dynamique même du totalitarisme :

Ainsi, personne ne vit sa propre réalité, écrit-il, mais seulement sa fonction sans faire l'expérience existentielle de sa vie, c'est-à-dire sans vivre son propre destin, qui pourrait être l'objet d'un travail — sur soi-même. L'horizon de l'homme fonctionnel n'est pas «le ciel étoilé» pas plus que «l'ordre moral qui sommeille en tout homme», mais les limites de son monde organisé: la réalité illusoire mentionnée plus haut. (Kertèsz, 2010,12.) 
Cette description phénoménologique de la langue totalitaire et de l'homme fonctionnel rejoint l'analyse que fait Piera Aulagnier de la notion d'aliénation dans Les destins du plaisir (Aulagnier, 1979, 37-52). L'originalité de Kertèsz consiste à décrire cette aliénation à partir d'une perspective linguistique.

L'aliénation est une tentation qui guette l'activité de pensée de tout Je: tel est le principe sur lequel repose l'analyse d'Aulagnier. Quelle est cette tentation? Retrouver la certitude en excluant autant le doute que le conflit. On peut la définir comme un phénomène intersubjectif dans la mesure où elle exige la rencontre d'un sujet avec un autre qui désire l'aliéner comme Freud le montre dans la relation du meneur à la masse.

Plus exactement avec un désir d'aliéner qui doit pouvoir trouver sur la scène sociale un autre sujet dont la pensée, l'action, induisent l'aliénation d'une partie ou de la totalité de ses semblables. (Aulagnier, 1979, 38)

C'est bien sur ce désir d'aliéner que repose, en partie, le phénomène totalitaire.

À titre d'illustration clinique, je citerai ce passage de Kertèsz dans son livre L'holocauste comme culture $(2009,114)$ :

Ceux qui ont vécu l'un des totalitarismes de ce siècle [...] partageront avec moi le souci de ce dilemme impossible à occulter. Parce que, dans la vie de chacun d'entre eux, il y a une période durant laquelle ils n'ont pas vécu leur propre vie, où ils se sont retrouvés dans une situation inconcevable, dans un rôle inexplicable pour le bon sens, où ils ont agi comme ils n'auraient jamais agi selon leur propre jugement, où ils ont été obligés de faire des choix qui leur étaient imposés non par une exigence interne, mais par une puissance extérieure cauchemardesque. C'est une période dont ils se souviennent ensuite confusément, voire avec confusion, ne s'y reconnaissent pas du tout, une époque qu'ils n'ont certes pas réussi à oublier totalement, mais qui s'est réduite avec le temps en une série d'anecdotes et qui de ce fait - c'est du moins ce qu'ils ressentent - n'est pas devenue une partie intégrante de leur personnalité, un vécu qui continue à la construire, bref, est restée une période qui n’a pas voulu se transformer en expérience.

Aux yeux de Kertèsz, lorsqu'on pense aux totalitarismes, le xxe siècle a comme trait distinctif d'avoir effacé, annihilé, balayé, pour utiliser son expression, 
toute personnalité chez le sujet, autrement dit d'avoir cherché à le désubjectiver. L'individu a été réduit à n'être qu'un individu de masse, anonyme, que rien ne distingue d'un autre individu. Toute différence a été abolie. Le dilemme qui se pose alors est le suivant : comment parvenir à établir une relation entre une personne et une personnalité formées par les expériences successives que vit le sujet, ce "processus éducatif et existentiel que les Allemands appellent Bildung» (Kertèsz, 2009, 114) et cette histoire qui, à chaque instant, nie et va jusqu'à anéantir toute personnalité? Il nous décrit comment a été vécu ce dilemme qui a introduit l'aliénation et même le meurtre d'âme au cœur du sujet en le contraignant à agir sous l'effet "d'une puissance extérieure cauchemardesque», tel un destin contre lequel il se sent totalement impuissant, et qui a fini par instaurer un clivage profond à l'intérieur du psychisme. La remémoration d'une telle période est source de confusion dans la mesure où ces sujets à qui le Pouvoir a assigné des emplacements inconcevables, contraires à tout ce que leur être le plus intime aspirait à occuper, ne se reconnaissaient pas dans ces emplacements, ni dans ces choix ni dans ces actions. Ils restent pris entre un refoulement qui n'a réussi qu'à demi et un clivage qui semble insurmontable, leur tentative de s'approprier ce passé échoue dans la mesure où les événements vécus dans ces périodes de leur histoire demeurent à jamais impossibles à transformer en expérience. La subjectivation échoue. J'exprimerai cela dans le langage de Bion en disant que la fonction alpha du sujet ne parvient pas à élaborer ni à transformer les éléments bêtas, ces faits non digérés, comme les appelle Bion - et dans notre cas impossibles à digérer - en éléments alphas, éléments digérés, mis au service de la pensée. Le processus de métabolisation ne peut pas avoir lieu. Nous savons quelles en sont les conséquences pour le nourrisson qui devient alors la proie d'une terreur sans nom. Je m'arrêterai sur l'expression sans nom qui qualifie la terreur, car le nom établit un lien entre un ensemble de phénomènes qui entretiennent entre eux des relations de succession et de contiguïté; il leur donne une cohésion et une signification, ce qui ne peut être le cas ici. Le vécu ne peut alors que devenir intolérable, le moi se fragmente et l'expérience apparaît comme dépourvue de toute signification. Bien des rescapés des camps ont vécu cette terreur jusqu'à la fin de leurs jours. Dans son dernier livre, Les naufragés et les rescapés, Primo Levi évoque une angoisse atavique qui peut être rapprochée de la terreur sans nom de Bion:

«celle dont on entend l'écho au deuxième verset de la Genèse: l'angoisse inscrite en chacun de nous du «tohu-bohu», de l'univers désert et vide, 
écrasé sous l'esprit de Dieu, mais dont l'esprit de l'homme est absent: ou pas encore né ou déjà éteint. $(1989,84)$

Est-ce possible, dans ce contexte, de retrouver sa langue personnelle, celle qui puisse dire sa tragédie, se la réapproprier? se demande Kertèsz. Cela prendra très longtemps, si c'est toutefois possible, répond-il. Cette réappropriation peut confronter l'individu au caractère indicible, ineffable de cette tragédie. L'auteur rattache cette réflexion à son enfance et à une expérience particulière qui l'a fait beaucoup souffrir mais qu'il n'était pas en mesure de comprendre:

«J'avais l'impression que je participais à un grand mensonge universel, mais que ce mensonge était en fait la vérité et que c'était de ma faute si j’y voyais un mensonge. (Kertèsz, 2009, 214)

Ce grand mensonge était la montée du fascisme et de son idéologie antisémite dans la Hongrie de son enfance dans les années trente. Ce qu'il ne saisissait pas alors et qu'il comprendra par la suite c'est que cette expérience était de nature linguistique. Cette expérience était « une protestation instinctive contre la société pro et pré-fasciste de Budapest des années trente qui me suggérait d'accepter comme un destin normal le danger qui me guettait.» (Kertèsz, 2009, 214)

Le sujet risque alors d'être condamné à rester prisonnier de cette pseudo réalité dont parle Kertèsz. Quel rapport pourrait-elle alors entretenir avec le langage? Ne pourrait-on pas y voir, à la limite, une création du langage?

C'est là que nous pouvons observer le pouvoir du langage, particulièrement celui du novlangue, tel que le Pouvoir absolu le manipule en faisant soit exister, grâce à lui, des réalités imaginaires soit en réduisant à néant des réalités existantes. Il établit alors un partage " entre l'être et le non-être, entre ce qui est et ce qui n'est pas ou ne peut pas être, faute d'un langage pour le dire.» (Dewitte, 2007, 12)

Qu'a été, par exemple, la langue communiste? Nous pourrions sans doute lui appliquer certaines des analyses que Klemperer fait de la langue allemande. Cependant, elle a eu d'autres dimensions. Le poète et essayiste polonais, Alexander Wat, qui a analysé la langue communiste, en a dégagé le cœur qu'il nomme la «sémantique stalinienne». Elle consiste, comme le précise Dewitte, à imposer « une représentation idyllique de la réalité [...] par le langage» (Dewitte, 2007, 212), et, surtout, à obliger «les hommes à dire que cette représentation idéale était leur réalité vécue, mais encore à le croire 
vraiment leur imposant ainsi un dédoublement schizophrénique permanent» (Dewitte, 2007, 212).

Wat parle «d'une gigantesque entreprise de corruption du langage humain.» (cité par Dewitte, 2007, 212)

Or cette langue est bien celle dont parle Celan dans l'Entretien dans la montagne:

Une langue en usage ici, $[\ldots]$ une langue pas pour toi et pas pour moi $[\ldots]$ une langue, de toujours, sans Je et sans Toi, rien que Lui, rien que Ça, comprends-tu, Elle simplement et c'est tout. (Kertèsz, 2009, 215)

C'est la langue des autres, commentera Kertèsz, «une langue qui est la conscience d'une société qui fonctionne comme si de rien n'était, une langue où l'exclu restera toujours un cas particulier, une pierre d'achoppement, un étranger : lui, eux ou ça, une langue qui a définitivement scellé l'exclusion propre à Auschwitz après Auschwitz. » (Kertèsz, 2009, 215)

Pour Kertèsz, le survivant ou quiconque veut parler de l'Holocauste affronte ce problème: ou bien s'intégrer à langue du pays où il vit et accepter ses conventions linguistiques en acceptant «les mots "victime", "persécuté", "survivant", etc., ainsi que le rôle et la conscience qu'ils impliquent, » ou se rendre compte peu à peu de son isolement et finir par baisser les bras. (Kertèsz, 2009, 216)

Il en conclut qu' "avec le temps, le poids insupportable de l'Holocauste a façonné les formes linguistiques du discours sur l'Holocauste qui abordent en apparence l'Holocauste alors qu'elles n'en effleurent même pas la réalité » (Kertèsz, 2009, 216).

Autrement dit, la parole qui dit l'Holocauste est elle même prise au piège de cette langue façonnée par les totalitarismes. Sa critique s'adresse d'abord au mot lui-même, «Holocauste», qui, dit-il, « est devenu un terme générique sacré servant à désigner la routine des massacres quotidiens, du gazage, des meurtres, de la solution finale, de la destruction des hommes » (Kertèsz, 2009, 216),

Il dénonce ceux qui veulent parler d'Auschwitz et le reconstruire avec la langue d'avant Auschwitz comme si aucune rupture ne s'était produite, comme "si la barbarie ne l'avait pas cassée » (Kertèsz, 2009, 216-217), comme si Auschwitz n'avait été qu'un accident, qu'un «déraillement» de l'histoire qui a fini, heureusement, par retrouver sa voie.

Il dénonce enfin impitoyablement ceux qu'il appelle les voyeurs de l'Holocauste, dont le cinéaste américain Steven Spielberg, pour qui 
l'Holocauste s'inscrirait « dans la continuité de la martyrologie millénaire du peuple juif et, en enjambant les monceaux de cadavres, la ruine de l'Europe et l'effondrement de toutes les valeurs, glorifient l'éternelle survie avec des images en couleur et une musique triomphale.» (Kertèsz, 2009, 217)

Cela vient confirmer à ses yeux ce que Tadeusz Borowski et Jean Améry écrivaient déjà lorsque le premier déclarait que « [1]a clameur des poètes, des avocats, des philosophes et des curés étouffera nos voix » (Kertèsz, 2009, 217), et le second, en écho: «Le fait que certains d'entre nous soient malgré tout restés en vie sera considéré comme un incident technique, un accident» (Kertèsz, 2009, 217)

Les véritables survivants et les véritables problèmes de la survie ne semblent intéresser personne. Pour Kertèsz ces deux auteurs «nous ont transmis leur véritable expérience de l'Holocauste » et "parlent déjà la langue d'après Auschwitz». (Kertèsz, 2009, 217) Mais quelle est cette langue? Kertèsz lui a forgé un nom à l'aide d'un terme musical: une langue atonale.

Je la qualifierais comme une langue en rupture, une langue dont la continuité a été brisée comme l'a été la continuité des vies des survivants. Il décrit le drame de ces survivants qui ont consacré leur vie à témoigner de l'Holocauste: ils n'ont pu ni accepter de vivre selon ce que la société leur proposait ni formuler leur expérience dans la langue d'avant Auschwitz:

Au lieu de s'efforcer d'oublier, de rechercher la chaleur d'une existence humaine normale, elles [ces personnes] reconstruisaient leur personnalité anéantie dans les camps d'anéantissement avec les expériences qu'elles avaient vécues dans ces camps: elles devenaient les médiums d'Auschwitz. (Kertèsz, 2009, 217-218)

Ils ont vite découvert «l'impossibilité de la survie».

«L'esprit d'Auschwitz s'était insinué en elles [ces personnes] comme un poison, ainsi que l'indifférence bien intentionnée de la société.» (Kertèsz, 2009, 218)

C'est ce qui, selon lui, aurait conduit au suicide Jean Améry, Tadeusz Borowski, Primo Levi et d'autres qu'il qualifie de « destins cohérents ». Il ressent pourtant un embarras en pensant à eux : comment, lui, a-t-il échappé au suicide? Dans son Journal de galère, en 1991, il fournit une analyse qui, en 2000, lui semble toujours exacte. De façon tout à fait paradoxale, la société communiste, cette société qui lui garantissait la continuité d'une vie de prisonnier excluant ainsi toute possibilité d'erreur, le sauva du suicide. Il ne fut 
pas atteint par la vague de désillusion qui commençait d'atteindre des personnes qui avaient partagé les mêmes expériences que lui mais vivaient dans des sociétés plus libres. Elles ont eu beau fuir en pressant le pas, la marée finit par les rattraper jusqu'à les noyer. Sa vie dans la Hongrie communiste était « une sorte de présent prolongé d'Auschwitz. L'Holocauste et la situation existentielle dans laquelle j'ai écrit sur l'Holocauste se sont inextricablement mêlés.» (Kertèsz, 2009, 219)

\section{Kertèsz écrivain}

Sa liberté d'écrivain était illimitée d'autant plus que la «publication était aussi incertaine que la survie de l'auteur» (Kertèsz, 2009, 219).

Il écrivait dans des conditions de censure totale, et cette oppression était créatrice de liberté, «la niant jour après jour et rendant jour après jour son existence d'autant plus évidente» (Kertèsz, 2009, 219). Même si ces considérations ne pouvaient que conduire au désespoir, puisqu'elles se heurtent à une impasse, Kertèsz pense qu'au fond de ce désespoir on peut voir briller une promesse, cet espoir au-delà de tout espoir dont parle Kierkegaard. Il réside dans la communauté de destin ou plus précisément dans la privation de destin. Écrire sur l'Holocauste était aussi pour lui une façon de nourrir l'illusion qu'il parlait de la dictature communiste et qu'en parlant de la souffrance infligée par les nazis, il parlait de la souffrance de tous, il en devenait leur porte-parole.

Cette illusion se nourrit du silence ou du refus avec lesquels les détenteurs du pouvoir reçoivent l'œuvre, et de l'isolement auquel ils réduisent l'auteur. (Kertèsz, 2009, 219)

S'il est vrai comme l'affirme Nietzsche que ce qui ne nous tue pas renforce notre vitalité, Kertèsz pense que la dictature non seulement lui a servi à rester en vie mais l'a aussi aidé à "trouver la langue dans laquelle je devais écrire» (Kertèsz, 2009, 220). Dans un régime totalitaire la langue n'est "pas pour toi et pas pour moi" pour reprendre l'expression de Celan, il n'existe pas de Je ni de Tu, mais un Nous «mystique et menaçant qui cache on ne sait qui» (Kertèsz, 2009, 220). Quel est alors le but de l'écrivain? Kertèsz le qualifie à la fois de négatif et de créatif:

s'extraire de la langue «en usage ici» qui a emprisonné tout sentiment et toute pensée les contraignant à la servir comme des bagnards; et c'est avec 
ce qui reste de la langue, avec quelques débris, qu'il doit créer son personnage, le survivant de l'Holocauste, lequel, pour citer Cioran, n'appartient plus à l'humanité. (Kertèsz, 2009, 220)

Kertèsz vit un drame linguistique au niveau de son écriture. Il reste attaché à sa langue maternelle; il l'aime. Dans un discours prononcé à Munich en 1996 sur le thème Patrie et pays (Kertèsz, 2009,), il écrit:

Il existe un pays où je suis né, dont j'ai la citoyenneté et, surtout, dont la langue merveilleuse est celle dans laquelle je parle, lis et écris mes livres; mais ce pays n'a jamais été le mien, c'est plutôt moi qui lui appartiens et, durant quatre décennies, il a été pour moi plus une prison qu'une patrie. (Kertèsz, 2009, 145. Ce sont mes italiques)

Malgré cet amour, il n'a pas de sentiment d'appartenance à ce pays. De plus cette langue n'est pas neutre dans la mesure où elle est devenue une langue totalitaire marquée à la fois par l'expérience nazie et par l'expérience communiste. Kertèsz nous donne un indice de cette lourde imposition lorsqu'il affirme "qu'il faut s'extraire de la langue "en usage ici" » (mes italiques), ce qui est le signe de sa défiance. L'usage du verbe s'extraire - dans la traduction fait penser au fait qu'il se sent pris, emprisonné dans une langue transformée par quarante années de dictature communiste, une langue réifiée. Extraire fait penser au minerai qu'on extrait du sol et qu'on doit débarrasser de sa gangue. La gangue linguistique est constituée par tous ces éléments toxiques dont parlait Klemperer à propos de la langue allemande. Mais si Kertèsz parle de s'extraire, c'est aussi parce que le danger d'une telle langue est son intériorisation. Sa violence est là, personne n'y échappe. Le sujet se met à parler et à ressentir en fonction de la langue qu'il parle. En fait, elle parle en lui et par lui. À travers l'emploi du verbe s'extraire, Kertèsz semble suggérer un travail d'arrachement à la langue au terme duquel l'écrivain se retrouve avec des restes, des débris à partir desquels il devra reconstituer sa langue et retrouver son pouvoir créateur.

À qui est destinée cette langue reconstituée? Tout dépend de la conception que l'on se fait de l'Holocauste. Est-ce un événement qui ne concerne que l'histoire juive? Est-ce la seule tragédie des juifs? Ce n'est pas la conception de Kertèsz. À ceux qui pensent qu'il y a une tendance à vouloir déposséder les juifs du vécu de l'Holocauste, il répond en soulignant que 
l'Holocauste [...] [est] une expérience universelle, un traumatisme européen. Auschwitz ne s'est pas produit hors de l'espace et du temps, mais dans la culture occidentale, dans la civilisation occidentale, et cette civilisation est une survivante d'Auschwitz au même titre que les quelques dizaines ou centaines de milliers d'hommes et de femmes disséminés dans le monde entier qui ont vu les flammes des crématoires, qui ont senti l'odeur de la chair humaine brûlée. Ces flammes ont anéanti toutes les valeurs européennes que nous estimions jusqu'alors, et à ce point zéro de l'éthique, dans ces ténèbres morales et intellectuelles, le seul point de départ possible est ce qui a produit ces ténèbres, à savoir l'Holocauste (Kertèsz, 2009, 221).

Pourtant son pays natal, la Hongrie, dénie tout cela en le marginalisant, en le caractérisant de phénomène périphérique. Ce qui amène Kertèsz à affirmer qu'il écrit ses livres dans une langue d'emprunt « qui, par sa nature, les rejette ou tout au plus les tolère à la périphérie de sa conscience.» (Kertèsz, 2009, 223) Kertèsz vit cela avec une certaine amertume d'autant plus qu'il aime sa langue maternelle. Il minimise l'impact de ce rejet lorsqu'il écrit :

Plus je suis étranger à la langue, plus je me sens fidèle à moi-même et à mon propos. J'aime écrire en hongrois, car cela me fait mieux ressentir l'impossibilité d'écrire. (Kertèsz, 2009, 223)

Il compare sa situation à celle de Kafka qui analyse, dans une lettre à Max Brod, la situation de l'écrivain juif et formule une série de paradoxes de l'impossibilité. Il " parle de trois impossibilités: celle de ne pas écrire, celle d'écrire en allemand, celle d'écrire autrement.» (Kertèsz, 2009, 223)

À quoi Kertèsz répond: "Je pourrais presque y ajouter une quatrième impossibilité, celle d'écrire. Aujourd'hui, il préciserait peut-être qu'il est impossible d'écrire sur l'Holocauste.» (Kertèsz, 2009, 223)

Quel est alors le statut de l'écrivain de l'Holocauste?

L'écrivain de l'Holocauste est partout et dans toutes les langues un réfugié de l'esprit qui formule toujours dans une langue étrangère sa demande d'asile intellectuel. (Kertèsz, 2009, 224)

La langue dans laquelle il s'exprime demeure toujours, pour Kertèsz, une langue étrangère. Il est un réfugié de l'esprit qui fait une demande d'asile 
intellectuel. C'est quelqu'un qui trouve refuge dans une autre langue, dans une autre culture parce que il est menacé dans sa culture d'origine. Sa demande d'asile est demande d'une culture et d'une langue où puisse être accueillie sa parole. C'est un exilé de l'esprit qui ne peut connaître

qu'un seul véritable problème, celui de l'émigration. Mais il ferait mieux de parler d'exil plutôt que d'émigration. De l'exil de sa seule véritable patrie, laquelle n'a jamais existé. Car, si elle existait, il ne serait pas impossible d'écrire sur l'Holocauste, l'Holocauste aurait alors une langue et l'écrivain de l'Holocauste pourrait s'insérer dans une culture existante. Mais elle n'existe pas. (Kertèsz, 2009, 224)

L'expérience hongroise est ici déterminante pour comprendre les paroles de Kertèsz. Il en conclut que

toute langue, tout peuple, toute civilisation, a un Je dominant qui perçoit le monde, l'ordonne et le représente. Ce Je collectif en action permanente est un sujet avec lequel une grande collectivité - nation, peuple, culture - peut d'une manière générale s'identifier, avec plus ou moins de succès. Mais où est la patrie de la conscience de l'Holocauste, quelle langue pourrait prétendre être le Je dominant de l'Holocauste, la langue de l'Holocauste? (Kertèsz, 2009, 224)

Il pose la question de la langue de l'Holocauste: est-il imaginable que l'Holocauste ait sa propre langue?

Si oui, cette langue ne devrait-elle pas alors être terrible et sinistre au point d'anéantir ceux qui la parleraient? (Kertèsz, 2009, 224-225)

Nous sommes au cœur de ce que Kertèsz désigne comme la langue exilée.

David Benhaïm 900, Rockland, app. 309

Outremont, Québec $\mathrm{H} 2 \mathrm{~V} 3 \mathrm{~A} 2$ sigmundb@videotron.ca 


\section{Références}

ALTOUNIAN, J., 2000, La survivance, Traduire le trauma collectif, Inconscient et Culture, Paris, Dunod.

ANISSIMOV, M., 1996, Primo Levi ou la tragédie d'un optimiste, Paris, Lattès.

AULAGNIER, P., 1979, Les destins du plaisir, Paris, Le fil rouge, PUF.

AULAGNIER, P., 1984, L'apprenti-historien et le maître-sorcier, Paris, Le fil rouge, PUF.

BENVENISTE, É., 1966, Problèmes de linguistique générale, 1, Paris, Gallimard.

BENVENISTE, É., 1974, Problèmes de linguistique générale, 2, Paris, Gallimard.

La Bible, Ancien Testament I, 1956, Paris, Bibliothèque de la Pléiade, NRF, Gallimard.

BION, R, W., 1967, Réflexion faite, Paris, Bibliothèque de psychanalyse, PUF, 1983.

BION, R, W., 1962, Aux sources de l'expérience, Paris, Bibliothèque de psychanalyse, PUF, 1979.

CAHN, R., 1991, Du sujet, Revue française de psychanalyse, LV, 6, 1371-1490.

CAMON, F., 1987, Conversations avec Primo Levi, Paris, Le Messager, Gallimard, 1991.

CARASSO, F.,1997, Primo Levi, le parti pris de la clarté, Paris, Belin.

CASTORIADIS-AULAGNIER, P, 1975, La violence de l'interprétation, Paris, Le fil rouge, PUF.

CELAN, P., 1960, Le Méridien, Paris, Fata Morgana, 2008.

CELAN, P., 1983, Entretien dans la montagne, Paris, Verdier, 2001.

DEWITTE, J., 2007, Le pouvoir de la langue et la liberté de l'esprit. Essai sur la résistance au langage totalitaire, Paris, Michalon.

FREUD, S., 1921, Psychologie de foules et analyse du moi in Essais de psychanalyse, Paris, Payot, 1987.

HÄHNEL, C., MESNARD, PH., 2003, Entretien: Imre Kertèsz: écrivain et prix Nobel, Mouvements, $\mathrm{n}^{\circ}$ 25, 115-122.

KERTÈSZ, I., 1992, L'Holocauste comme culture, Paris, Actes Sud, 2009.

KERTĖSZ, I., 1992, Journal de galère, Paris, Actes Sud, 2010.

KLEMPERER, V., 1975, LTI, la langue du troisième Reich, Paris, Albin Michel, 1996.

LEVI, P., 1946, Rapport sur Auschwitz, Paris, Kimé, 2005.

LEVI, P., 1986, Les naufragés et les rescapés, Paris, Arcades Gallimard, 1989.

LEVI, P., 1958, Si c'est un homme, Paris, Julliard, 1987.

MARCUSE, H., 1964, L’homme unidimensionnel, Paris, Les Éditions de Minuit, 1968.

MARSALEK, 1977, Mauthausen, Milan, la Pietra.

MOSCOVICI, M., 2002, Le meurtre et la langue, Paris, Métailié.

ZALTZMAN, N., 1998, De la guérison psychanalytique, Paris, Épîtres, PUF. 